\title{
Pigment epithelium-derived factor in the monkey retinal pigment epithelium and interphotoreceptor matrix: apical secretion and distribution
}

\author{
S. Patricia Becerra ${ }^{\mathrm{a} * *}$, Robert N. Fariss ${ }^{\mathrm{b}}$, Yan Q. Wu ${ }^{\mathrm{a}}$, Luis M. Montuenga,d, \\ Paul Wong ${ }^{\mathrm{a}, \mathrm{e}}$, Bruce A. Pfeffer ${ }^{\mathrm{a}, \mathrm{f}}$

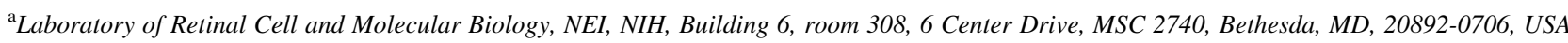 \\ ${ }^{\mathrm{b}}$ Laboratory of Mechanisms of Ocular Diseases, NEI, NIH, Bethesda, MD, USA \\ ${ }^{\mathrm{c}} \mathrm{DCS}, \mathrm{NCI}, \mathrm{NIH}$, Bethesda, MD, USA \\ ${ }^{\mathrm{d}}$ Department of Histology and Pathology, University of Navarra, Pamplona, Navarra, Spain \\ e Department of Biological Sciences, University of Alberta, Edmonton, Canada \\ ${ }^{\mathrm{f}}$ Bausch and Lomb, Rochester, NY, USA
}

Received 16 April 2003; accepted in revised form 29 October 2003

\begin{abstract}
Pigment epithelium-derived factor (PEDF) is an extracellular protein derived from the retinal pigment epithelium (RPE), a tissue formed by polarized cells that release growth and trophic factors in a directional fashion. We have investigated the distribution and directional release of PEDF protein by the monkey RPE. We established primary cultures of monkey RPE cells that expressed the $P E D F$ gene, and that synthesized and secreted the PEDF protein. Northern analysis of RPE cultures and monkey ocular tissues showed that $P E D F$ transcripts were highly expressed in RPE as compared with several other monkey ocular tissues, being even more abundant in cultured cells than they were in the native RPE. The differentiated RPE cells in culture secreted protein that shared the immunological, biochemical and biological characteristics of PEDF. The overall PEDF levels in the RPE conditioned media reached $6.5 \mathrm{mg} \mathrm{ml}^{-}$after 8 days in culture (i.e. $1.1 \mathrm{pg}$ of PEDF per RPE cell). RPE cells were cultivated on permeable supports as monolayers forming a barrier between apical and basal compartments. Apical and basal culture media were sampled at three or four-day intervals for 18 cycles, and the PEDF content was quantified. Most of the PEDF protein was significantly higher in the apical than in the basal medium ( $>4$ times) at the initial recovery intervals, to be detected only in the apical medium at the latter intervals. In the native monkey eye, the concentration of soluble PEDF in the interphotoreceptor matrix (144 nM) was 7-fold and 25-fold greater than in vitreous and aqueous, respectively. PEDF was abundant in the interphotoreceptor matrix surrounding rod and cone outer segments, and was detectable at lower levels in the RPE as visualized by confocal microscopy. We concluded that PEDF synthesized by the RPE is secreted preferentially from the apical surface and is distributed apically to the RPE bordering the outer segments of photoreceptors. PEDF can be a useful marker for RPE polarization and differentiation. The polarization of RPE may be an important mechanism to control PEDF secretion and our results offer interesting possibilities on regulation of PEDF.
\end{abstract}

(C) 2004 Elsevier Ltd. All rights reserved.

Keywords: retinal pigment epithelium; pigment epithelium-derived factor; secretion; polarization; interphotoreceptor matrix; monkey eye; apical; basal

\section{Introduction}

The retinal pigment epithelium (RPE), situated between the neurosensory retina and the vascularized choroid, maintains the photoreceptors and the choriocapillaris in

\footnotetext{
* Corresponding author. Dr S. Patricia Becerra, Laboratory of Retinal Cell and Molecular Biology, NEI, NIH, Building 6, room 308, 6 Center Drive MSC 2740 Bethesda, MD, USA.

E-mail address: becerrap@nei.nih.gov (S. Patricia Becerra).
}

the normal eye, and is involved in the pathogenesis of retinal degeneration and choroidal neovascularization in agerelated macular degeneration. It performs functions essential for photoreceptor survival and secretes factors important for homeostasis of the outer retina. Formed by cells that polarize, the RPE can secrete components in a directional fashion. For example, hyaluronan, matrix metalloproteinase-2 and tissue inhibitor of metalloproteinase-1 synthesized by the RPE are secreted preferentially from the apical surface (retina facing) toward the photoreceptors 
(Padgett et al., 1997; deS Senanayake et al., 2001), while tissue inhibitor of metalloproteinase-3, fibroblast growth factor 5, interleukin-6 and interleukin- 8 are secreted from the basolateral surface of these cells facing the choroid (Fariss et al., 1997; Dunn et al., 1998; Holtkamp et al., 1998). Its polarization is under constant regulation by signaling molecules coming from the choroid and the retina or extracellular stimuli. For example, vasoactive intestinal peptide, a 28-amino acid neuropeptide and differentiation promotor during development of the functional RPE, stimulates macromolecule secretion at the apical surface and fluid transport from the apical to the basal surface of the RPE sheet (Koh, 2000); oxygen or extracellular matrix components from RPE modulate the polarity of secretion of angiogenic factors, such as vascular endothelial growth factor, from the RPE (Mousa et al., 1999; Blaauwgeers et al., 1999). Thus the polarity of the RPE plays a role in maintaining a balance of secreted factors essential for the functional RPE.

One component secreted by the RPE is pigment epithelium-derived factor (PEDF), a protein with neurotrophic and antiangiogenic activities expressed mainly in compartments of the eye. PEDF acts in neuronal survival and differentiation on photoreceptor and neuronal cells of the retina and central nervous system (Becerra, 1997; Cayouette et al., 1999; Houenou et al., 1999; Cao et al., 2001; Crawford et al., 2001). It is also a major inhibitor of neovascularization and is responsible for excluding vessels from invading the retina, vitreous and cornea (Dawson et al., 1999; Stellmach et al., 2001; Bouck, 2002). These biological activities are of great importance for the development and maintenance of normal physiological functions in the eye. Biochemical and immunochemical studies have identified PEDF as a soluble extracellular protein localized in washes of the interphotoreceptor matrix (IPM), vitreous and aqueous of several mammalian species (Tombran-Tink et al., 1995; Wu et al., 1995; Ortego et al., 1996; Alberdi et al., 1998; Karakousis et al., 2001). PEDF, discovered as a product secreted by cultured RPE cells from fetal human eyes (Tombran Tink, 1991), can be released from a variety of cells, including stably transfected eukaryotic cells with full length $P E D F$ cDNA under the control of recombinant transcriptional promoters (Stratikos et al., 1996; Perez-Mediavilla et al., 1998). By sequence homology, PEDF is a member of the serpin superfamily (Steele et al., 1993), formed by proteins with a common overall protein conformation with a variety of biological functions, most of which are extracellular serine protease inhibitors. Like serpins, PEDF is a globular and compact protein of $\sim 50 \mathrm{kDa}$ that is highly resistant to proteolytic cleavage except for an exposed loop towards its carboxylend; however, it does not behave as an inhibitor of proteases (Becerra et al., 1995). It has binding affinity for components of extracellular matrixes, such as glycosaminoglycans (Alberdi et al., 1998) and collagens (Meyer et al., 2002), which represents its molecular association with the IPM, and other extracellular matrixes. More interestingly, PEDF has binding affinity for receptors on the surface of cells from retina and the CNS known to respond to its stimuli, such as retinoblastoma cells, cerebellar granule cell neurons, photoreceptors, motor neurons (Alberdi et al., 1999; Aymerich et al., 2001; Bilak et al., 2002). While the deposition of PEDF in extracellular ocular compartments coincides with their avascular nature, the discrete distribution of PEDF binding sites in the bovine retina to the inner segments of photoreceptors (Aymerich et al., 2001) is consistent with PEDF's in vivo effects on survival of photoreceptors (Cayouette et al., 1999; Cao et al., 2001).

Given the neurotrophic and antiangiogenic activities of PEDF and its potential exploitation as a therapeutic agent for retinal diseases triggered by photoreceptor degenerations and abnormal neovascularization, it is of interest to determine its levels and distribution in the RPE/retina for physiological studies and development of therapeutics. Using the monkey eye as a non-human primate model, we have (1) compared the levels of $P E D F$ gene expression among ocular tissues; (2) determined PEDF protein levels in extracellular compartments of the eye; (3) characterized in detail the secreted monkey PEDF protein; and (4) investigated a directional delivery of PEDF from RPE in culture and in situ. We discuss the implications of a polar secretion and distribution of PEDF by the RPE on the retina.

\section{Materials and methods}

\subsection{Tissue samples}

Eyes were from juvenile Rhesus monkeys (aged 3-5 years) using procedures in strict compliance with the NIH Guidelines for Care and Use of Laboratory Animals. Eyes were processed for immunolabeling or ocular tissues dissected while chilled on ice. Aqueous and vitreous extracts were obtained as described by $\mathrm{Wu}$ and Becerra (1996). IPM lavages were obtained after dissection of the anterior portion, removal of vitreous and retina ( $\mathrm{Wu}$ et al., 1995), or alternatively, by cannulation (Pfeffer et al., 1983), a method known to assure an IPM lavage free of intracellular contamination maintaining the topological integrity of the IPM compartment (Adler, 1989).

\subsection{RPE cell culture}

Confluent first passage Rhesus monkey RPE was utilized to generate conditioned media samples and for extraction of total RNA. The methodology used for establishing primate RPE cultures was as described in detail by Pfeffer (1990). In summary, cells were maintained as stable monolayers in 12well clusters for at least two weeks and then experiments were performed after cultured cells had achieved biochemical and physiological markers of the differentiated tissue in vivo. These markers include de novo melanization, 
formation of domes (emblematic of vectorial fluid transport), morphological apical-basal polarization, and expression of cellular retinaldehyde-binding protein. Confluent wells contained $6 \times 10^{5}$ cells each, as calculated from hemocytometer counting of parallel cultures. For production of conditioned media, RPE cells were maintained in proteinfree defined medium (Pfeffer, 1990) modified as follows: (1) serum, bovine retinal extract, insulin and transferrin were omitted, (2) Albumax was replaced by Chemically Defined Lipid Concentrate $(0 \cdot 1 \%(\mathrm{v} / \mathrm{v})$; Gibco-BRL, Gaithersburg, MD, USA); and (3) $0.05 \%$ (w/v) each of dimethyl betacyclodextrin and hydroxypropyl beta-cyclodextrin (Cyclodextrin Technologies Development, High Springs, FL, USA) were added The monolayer was washed twice with proteinfree medium, and then $0.75 \mathrm{ml}$ were added per well. After the third and sixth days, glucose, fructose, essential amino acids, and vitamins were supplemented to the cultures, along with water to correct for volume loss by evaporation. Throughout the eight-day period, the $\mathrm{pH}$ of the medium remained constant and cell morphology was unchanged. Medium was collected after eight days in culture, immediately frozen in dry ice and stored at $-80^{\circ} \mathrm{C}$.

\subsection{Culture of RPE Cells on permeable supports}

To investigate the polarization release of PEDF, RPE monolayers were established in complete medium (Pfeffer, 1990) on $9 \mathrm{~mm}$ diameter cell culture inserts containing $0.4 \mu \mathrm{m}$ pore size polyethylene terephthalate membranes (Cyclopore, Falcon, Lincoln Park, NJ, USA). Cultures were monitored visually under an inverted microscope to assess cell proliferation and morphology at confluence. Upon seeding in the inserts, the RPE cells displayed low levels of pigmentation. A stable, confluent monolayer was attained by 12 days and the culture medium was replaced in both chambers with fresh medium. After that, the harvesting of medium from apical $(0.5 \mathrm{ml})$ and basal $(1.2 \mathrm{ml})$ compartments, and its replenishment were repeated in cycles of alternating 4 and 3 days. At 19 days following seeding, de novo pigmentation was noticeable in the cell and was retained during the remaining 15 cycles of medium exchanges. Conditioned media were stored at $-80^{\circ} \mathrm{C}$. Similar preparations have been shown, with the use of a Millicell-Electrical Resistance System (Millipore, Bedford, MA, USA), to develop a transepithelial resistance in the range of $30-50 \mathrm{ohm}-\mathrm{cm}^{2}$.

\subsection{Northern blotting}

The method used was as described in Wong et al., 2000. Briefly, individual ocular tissues were dissected from twenty juvenile monkey eyes and their RNA extracted for northern blot analysis. Blots were probed with a ${ }^{32} \mathrm{P}$ radiolabeled human PEDF cDNA fragment. Total RNA from each tissue was pre-stained with ethidium bromide prior to electrophoresis and gels were photographed after electrophoresis. The relative levels of PEDF mRNA for each tissue were calculated as the ratio of densitometric intensity of the PEDF divided by the combined $18 \mathrm{~S}$ and $28 \mathrm{~S}$ RNA intensities (indicated as 'ratio').

\subsection{Western blotting and quantitation of PEDF protein}

The methods used have been described before by $\mathrm{Wu}$ et al. (1995). Briefly, western transfers were immunoreacted with Ab-rPEDF with an enzymatic colorimetric method for detection. Alternatively, a monoclonal antibody to PEDF (MAB1059; Chemicon) was used, as described before (Meyer et al., 2002). Quantitation was performed by densitometric scanning of immunoreactive stains and relative to those of known amounts of bovine or human PEDF protein as standards. Quantitation of total protein was performed using a BioRad Protease Assay kit (BioRad).

\subsection{PEDF protein purification}

To optimize a purification scheme for PEDF from monkey RPE conditioned media and vitreous extracts we modified previous methods for the purification of bovine PEDF (Wu et al., 1995; Wu and Becerra, 1996). These methods involved ammonium sulfate fractionation, cationexchange column chromatography and size-exclusion column chromatography. In contrast with the previous methods, monkey PEDF did not partition into one fraction by ammonium sulfate saturation, not even with $90 \%$ of the salts (unpublished observations). Therefore, for recovery purposes, concentration by ammonium sulfate precipitation was avoided for PEDF from monkey sources. The protein was purified to near homogeneity by two purification steps. First, proteins were fractionated by S-Sepharose column chromatography as described (Wu et al., 1995). PEDFcontaining fractions were then pooled and subjected to sizeexclusion ultrafiltration using Centricon-100 to exclude large molecules and the filtrate applied to centricon-30 to exclude small molecules and concentrate the protein. The final sample yielded a highly pure protein at $1 \mu \mathrm{g}$ PEDF per $\mathrm{ml}$ of monkey vitreous, and 2.6 $\mu \mathrm{g}$ PEDF per ml media with a $40 \%$ recovery. We have observed that the recovery and purity of the final PEDF sample decreases with increasing concentration of serum in the medium (Wu and Becerra, personal observations). In a similar fashion, PEDF was purified from IPM lavages and aqueous extracts. However, the purity was lower than for vitreous and the RPE conditioned media, e.g., $0.5 \mu \mathrm{g}$ of PEDF in $115 \mu \mathrm{g}$ of total protein from IPM lavages from three eyes. The final samples were stored at $-80^{\circ} \mathrm{C}$.

\subsection{Controlled proteolysis}

To examine the overall protein conformation of the purified protein we performed the typical assay for serpin proteins, controlled proteolysis, as described (Becerra et al., 
1995). Briefly, purified monkey PEDF protein was mixed with limited concentrations of chymotrypsin in a $15 \mu \mathrm{l}$ reaction containing $20 \mathrm{mM}$ Tris $\mathrm{HCl}, \mathrm{pH} 7 \cdot 5,150 \mathrm{~mm} \mathrm{NaCl}$, $1 \mathrm{~mm}$ EDTA, and incubated at $25^{\circ} \mathrm{C}$ for the indicated periods of time. The reactions were stopped by addition of SDS-PAGE sample buffer and freezing in dry ice. After heating at $100^{\circ} \mathrm{C}$ for $3-5 \mathrm{~min}$, the reaction mixtures were subjected to SDS-PAGE to compare migration patterns of the products. A parallel set of samples was subjected to $\mathrm{N}$ terminal sequence determination of peptides using an Applied Biosynthesis model 477 sequencer (Foster City, CA, USA) following the manufacturer's protocols.

\subsection{Biological assay for PEDF}

Neurite outgrowth from retinoblastoma cells was as described by Becerra et al. (1993). Briefly, human Y-79 cells exponentially growing in serum-containing medium were washed twice with phosphate buffered saline and plated $\left(1.25 \times 10^{5}\right.$ cells per $\left.\mathrm{ml}\right)$ in serum-free minimal essential medium supplemented with a mix of insulin, transferrin and selenium. Effectors were then added to the cultures. After 7 days at $37^{\circ} \mathrm{C}$ in $\mathrm{CO}_{2}$, cells were attached to poly-D-lysine coated plates with fresh serum-free medium. The differentiation state of the cultures was monitored at various intervals after attachment by light microscopy.

\subsection{Immunolabeling}

For immunofluorescence, following enucleation, monkey eyes were dissected and fixed by immersion for $4 \mathrm{hr}$ in $4 \%$ formaldehyde in PBS pH 7.3. Tissue was stored in $0.5 \%$ formaldehyde in PBS at $4{ }^{\circ} \mathrm{C}$. Retina/RPE/choroid samples were excised and washed in PBS and then embedded in 7\% agarose in PBS according to Hale and Matsumoto (1993). A Leica vibrating microtome was used to cut $100 \mu \mathrm{m}$ thick slices of tissue for immunofluorescence. Sections were blocked briefly in 5\% normal goat serum in PBS and then immunolabeled with a monoclonal antibody to PEDF (Chemicon) diluted 1:100 in ICC buffer (PBS with $0.5 \%$ BSA, 0.2\% Tween-20, $\mathrm{pH} 7.3$ ) and incubated overnight at $4^{\circ} \mathrm{C}$. Biotinylated peanut agglutinin (PNA) diluted 1:200 (Vector Labs) and streptavidin Cy5 diluted 1:200 (Jackson ImmunoResearch) were used to label cone matrix sheaths. Alexa568 phalloidin (Molecular Probes) was diluted 1:80 and used to label filamentous actin. Sections were washed repeatedly and labeled in the dark for $4 \mathrm{hr}$ with the nuclear dye DAPI (4',6-diamidino-2-phenyl-indole, dihydrochloride) (Molecular Probes) $\left(1 \mu \mathrm{g} \mathrm{ml}^{-1}\right)$ and secondary antibody -goat anti-mouse Cy3 (Jackson ImmunoResearch) or goat anti-mouse Alexa488 (Molecular Probes). Following repeated washing, sections were mounted in Gel Mount (Bio-Meda) and protected with a coverslip. Primary antibody was omitted from sections used as negative controls Specimens were analyzed on a Leica SP2 laser scanning confocal microscope equipped with Nomarski optics. Samples were scanned in sequential scan mode to reduce bleed-through artifacts. PEDF-immunolabeled and negative control sections were imaged under identical scanning conditions. Files were imported into Photoshop and converted to psd format for layout purposes.

For immunohistochemistry the fixed monkey retina sections were immunoreacted with a polyclonal antiserum to human recombinant PEDF Ab-rPEDF (Wu et al., 1995) and detection was by the peroxidase-mediated diaminobenzidine (DAB) precipitation method was as described before (Montuenga et al., 1997). Negative controls were immunoreacted without primary antibody or with preabsorbed AbrPEDF. Ab-rPEDF was preabsorbed by incubating the antiserum with PEDF protein covalently attached to resin $3 \mathrm{M}$-Emphazed (6 $\mathrm{mg}$ rhuPEDF $\mathrm{ml}^{-1}$ resin, and prepared as described by Alberdi et al. (1999) for $16 \mathrm{hr}$ at $4^{\circ} \mathrm{C}$. The resin mixture was transferred to a disposable column and unbound antisera proteins were eluted by gravity flow and collected as preabsorbed Ab-rPEDF. Immunolabeling was visualized by light microscopy.

\section{Results}

\subsection{Distribution of PEDF gene expression and PEDF protein in monkey eyes}

To identify the major potential sites of PEDF protein synthesis we analyzed the distribution of $P E D F$ transcripts in the monkey eye. We established primate RPE cultures and utilized confluent first passage Rhesus monkey RPE. Northern blot analyses of individual ocular monkey tissues and cultured RPE cells detected PEDF transcripts of $1.5 \mathrm{~kb}$, in agreement with the size for the human PEDF mRNA (Steele et al., 1993; Pignolo et al., 1993), detected in all tissues tested at different levels (Fig. 1). Expression was the highest in trabecular meshwork and RPE, followed by sclera, ciliary body, orbital muscle and retina, and the lowest in iris, limbus and cornea, based on densitometry. The levels of PEDF mRNA in the cultured RPE cells were $>3$-fold higher than in the RPE/choroid tissue, and both $>5$-fold higher than in retina.

Because PEDF is an extracellular protein we compared the protein obtained from the culturing medium of RPE cells and ocular fluids IPM, vitreous and aqueous. Western analysis of all the samples with monoclonal or polyclonal antibodies to PEDF revealed immunoreactivity migrating as a protein of $50 \mathrm{kDa}$ (Fig. 2), which was blocked when challenged with recombinant human PEDF protein (data not shown) indicating specific immunoreactivity for PEDF in monkey RPE conditioned medium, IPM, vitreous and aqueous. PEDF-immunoreactive bands migrating with slightly different speeds for vitreous and aqueous in Fig. 2(B) lanes 3 and 4 resemble two species of PEDF with differences in glycopeptides containing $\mathrm{N}$ acetylneuraminic acid (NeuAc) and $N$-acetylhexosamine 


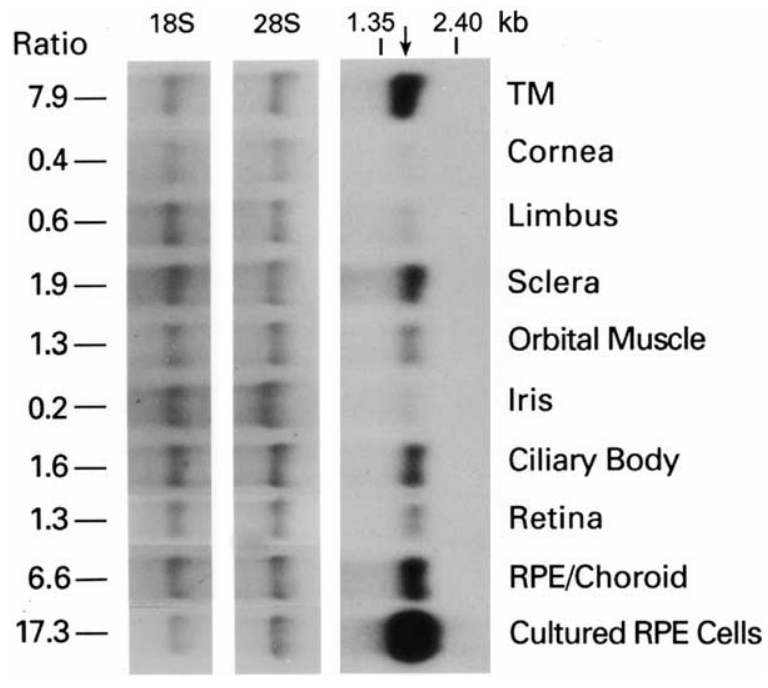

Fig. 1. Distribution of $P E D F$ gene expression in monkey eye tissues. Northern blot of RNA from monkey ocular tissues shows detection of hybridization of a ${ }^{32} \mathrm{P}$-radiolabeled human PEDF cDNA fragment (right panel). Arrow indicates the migration position of the PEDF transcript. Migration of size RNA markers is indicated at the top. Left and middle panels show ethidium bromide staining of the $28 \mathrm{~S}$ and $18 \mathrm{~S}$ RNA. The relative levels of PEDF mRNA per the combined 18S and 28S RNA intensities are indicated at the bottom as 'ratio'. TM corresponds to trabecular meshwork.

(HexNAc), as reported by Duh et al. (2002). However, an apparent single band was observed when a gradient polyacrylamide gel was used (see Fig. 2(C), lane 2). A summary and analysis of the purification of monkey PEDF from RPE conditioned media and vitreous are shown in Table 1 and Fig. 2(A). PEDF-immunoreactivity of 50-kDa was detected in monkey IPM lavages only after separation of PEDF from other IPM proteins by cationexchange column chromatography (Fig. 2(B); lanes 1 and 2), suggesting that IPM proteins comigrating with PEDF might have obstructed the PEDF-antibody interaction in westerns of the total IPM lavage. Similarly, PEDF was detected in western blots of monkey retina and RPE tissue extracts only after cation-exchange chromatography (Fig. 2(C)). PEDF was estimated in the IPM after purification by cation-exchange column chromatography and in the aqueous humor. The concentrations and amounts of PEDF in monkey IPM, vitreous and aqueous were calculated and are summarized in Table 2.

\subsection{Characterization of monkey PEDF protein}

We then characterized the purified monkey PEDF protein in detail. The size of the purified protein was determined by SDS-PAGE using several gels of a variety of polyacrylamide concentrations and under reducing conditions. The calculated size was $50700 \pm 1000-\mathrm{Mr}$ and is in agreement with the size for the native and recombinant bovine and human PEDF proteins (Wu et al., 1995, and Wu and Becerra, 1996; Stratikos et al., 1996; Perez-Mediavilla et al., 1998). The monkey PEDF behaved as a monomeric protein by size-exclusion ultrafiltration.

Controlled proteolysis of the purified protein with chymotrypsin rendered limited proteolytic products of $\sim$ 46-kDa (Fig. 3(A) and (B)). However, trypsin treatments did not produce a discrete limited product (data not shown). Edman degradation of the limited chymotryptic reactions
A

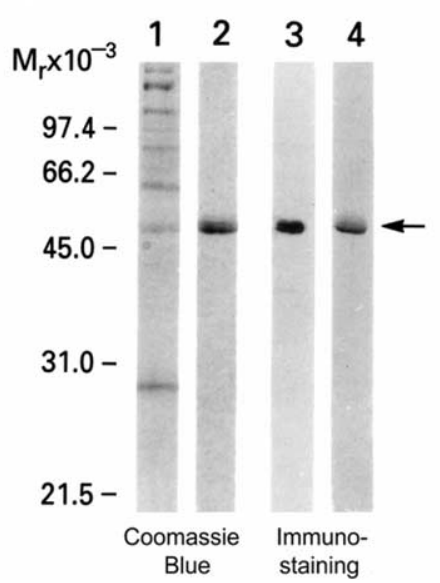

B

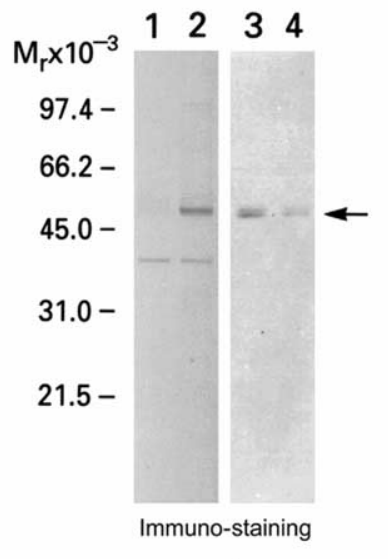

C

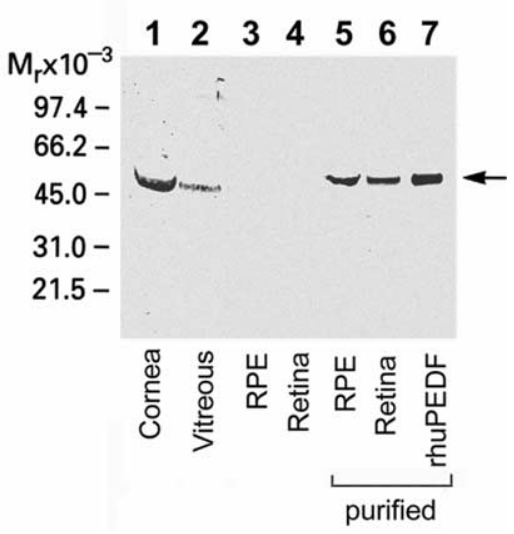

Fig. 2. Monkey PEDF in extracellular ocular compartments. (A) Secretion of PEDF from cultured RPE. Media from monkey RPE cells after 8 days in culture was collected and PEDF protein was quantified and purified. SDS/12.5\% PAGE followed with Coomassie Blue staining and immunoblotting with antiserum Ab-rPEDF are shown, as indicated to the bottom. Lanes 1 and 3 correspond to proteins from medium, and lanes 2 and 4 to the purified sample. (B) Western blots of ocular extracts showing immunoreactivity of antiserum Ab-rPEDF of a 50-kDa protein in the IPM, vitreous and aqueous of juvenile monkey eyes. SDS/10-20\% PAGE was for lanes 1 and 2; SDS/12.5\% PAGE was for lanes 3 and 4 . Lane 1 corresponds to IPM lavage concentrated 5-fold (20 $\mu$ ); lane 2 to IPM eluate of S-Sepharose column concentrated 10-fold (20 $\mu$ l); lane 3 to vitreous concentrated 4 -fold (5 $\mu 1)$; lane 4 to aqueous concentrated 5-fold (15 $\mu$ l). Arrow indicates the migration position of a 50-kDa PEDF protein. (C) Western blot of soluble tissue extracts before and after being subjected to S-sepharose column chromatography showing immunoreactivity of monoclonal antibody to PEDF. SDS/10-20\% PAGE of samples applied to lanes as indicated to the bottom of the panel, and rhuPEDF corresponds to purified recombinant human PEDF. PEDF in monkey IPM lavage, RPE and retina soluble extracts was detected only after S-sepharose column chromatography. Migration positions of SDS-PAGE molecular weight standards to the left of each panel. 
Table 1

Purification of monkey PEDF

\begin{tabular}{lllll}
\hline & $\begin{array}{l}\text { Total protein } \\
(\mathrm{mg})\end{array}$ & $\begin{array}{l}\text { PEDF protein } \\
(\mu \mathrm{g})\end{array}$ & $\begin{array}{l}\text { Yield } \\
(\mu \mathrm{g})\end{array}$ & $\begin{array}{l}\text { Recovery } \\
(\%)\end{array}$ \\
\hline RPE-CM $^{\mathrm{a}}$ & 44 & 650 & 260 & 40 \\
Vitreous $^{\mathrm{a}}$ & 4.5 & 65 & 25 & 38 \\
\hline
\end{tabular}

${ }^{\text {a }}$ Purification through S-Sepharose column chromatography followed by exclusion ultrafiltration using $30-100 \mathrm{kDa}$ limits. Starting material was $100 \mathrm{ml}$ for RPE conditioned media for 8 days (RPE-CM), and $65 \mathrm{ml}$ for vitreous (54 eyes).

revealed only one peptide sequence, TF?LDYHLNQPFIFVLRDT, which mapped to an internal region starting at Thr-383 of the human PEDF sequence. This polypeptide fragment corresponded to a $4 \mathrm{kDa}$ chymotryptic product from the carboxy-end of PEDF. Because sequencing of the intact protein did not yield an amino-terminal sequence, the 46-kDa polypeptide was assumed to correspond to the chymotryptic product from the amino-end of the PEDF. These observations imply a single chymotryptic cleavage site available in the monkey PEDF between serpin positions $\mathrm{P} 1-\mathrm{P} 1^{\prime}$ (382-383, human PEDF numbering), typical of human and bovine PEDF that have P1 = leucine amino acid residue (Becerra, 1997) (Fig. 3(B)).

Neurite-outgrowth assays corroborated that the monkey protein from RPE and vitreous has the typical neurotrophic activity on retinoblastoma cells (Fig. 3(C)-(E)) described for PEDF from human and bovine sources (Becerra et al., 1993; Wu et al., 1995).

Together these results demonstrate that the purified monkey protein (1) shared size and the overall protein folded conformation typical of PEDF, and serpins in general; (2) had identical amino acid sequence downstream from the P1 position to the human, bovine and mouse PEDF; (3) immunoreacted with specific antibodies to human PEDF; and (4) had the biological activity of PEDF. Thus the monkey RPE-secreted PEDF protein has similar biochemical, immunological, structural and functional characteristics to the naturally occurring PEDF of the monkey eye and those described previously for bovine and human PEDF proteins.

Table 2

PEDF protein in the monkey eye

\begin{tabular}{lllll}
\hline & $\begin{array}{l}\text { PEDF protein } \\
(\mu \mathrm{g} \text { per eye })\end{array}$ & $\begin{array}{l}\text { PEDF protein } \\
(\mu \mathrm{g} / \mathrm{ml})\end{array}$ & $\begin{array}{l}\text { PEDF } \\
\text { protein }(\mathrm{nM})\end{array}$ & $\begin{array}{l}\text { PEDF/Total } \\
\text { protein }(\%)\end{array}$ \\
\hline IPM $^{\mathrm{a}}$ & 0.45 & 7.2 & 144 & 0.14 \\
Vitreous $^{\mathrm{a}}$ & 1.2 & 1 & 20 & 1.4 \\
Aqueous $^{\mathrm{a}}$ & 0.06 & 0.3 & 5.7 & $\mathrm{nd}$ \\
\hline
\end{tabular}

a The estimated volume of IPM, vitreous and aqueous were 0.063, 1.2 and $0.2 \mathrm{ml}$, respectively. Starting material was three eyes for IPM, 54 eyes for vitreous and aqueous.

\subsection{Steady-state polarity of PEDF protein in cultured RPE cells}

A vectorial release of PEDF by cultured RPE was investigated. We cultivated monkey RPE cells to confluence on permeable supports to allow the epithelial cells to form a barrier between apical and basal medium compartments and to promote RPE polarization. Medium from both compartments was removed at intervals of 3-4 days and replaced with fresh medium. The conditioned medium was subjected to western blotting with antibodies to PEDF. As shown in Fig. 4, PEDF was detected in the apical media with levels of immunoreactivity remaining constant throughout a period of 63 days. In contrast, the levels of PEDF that accumulated in the basal media were lower than in the apical media and decreased during the first 42 days, after which it was virtually undetectable. During the initial phase of our study, the concentration of PEDF was to be estimated about 14 and $1.5 \mu \mathrm{g} \mathrm{ml}^{-1}$ in the apical and basal medium, respectively. Based on the intensity of the PEDF immunoreactivity in our western blots (Wu et al., 1995) the apical:basal ratio of PEDF was determined. Considering the volumes in each chamber, the apical:basal ratio started at 3.9:1 (i.e. $80 \%$ of the total PEDF was detected in the apical compartment) and it then increased to a greater ratio as PEDF levels were detected only in the apical compartment during the latter course of the study. To demonstrate that this pattern of secretion polarity was specific to PEDF, the distribution of another RPE secreted protein, TRPM-2/clusterin, was followed in the same cultures, and showed a different pattern, being preferentially distributed in the basal media compartment with an apical:basal ratio of 1:2 at the end of the 63-day sampling period, (Wong et al., 2000). These observations imply that the distribution of these individual proteins was maintained at ratios specific for each secreted macromolecule, and our results were not due to a passive process of general secretion into the medium by the RPE cells. Thus, the steady-state polarity of PEDF from RPE cells in culture indicates higher accumulations of PEDF to the apical side and suggests preferential secretion of PEDF from the apical membrane of RPE cells.

\subsection{Distribution of PEDF in the RPE-retina in situ}

We used confocal microscopy to examine the distribution of PEDF in the RPE/retina region of monkey eyes. Immunofluorescence of monkey retina with monoclonal antiserum to a carboxy-terminal end fragment of human PEDF, anti-PEDF, showed a strong and specific immunoreactivity in the IPM (Fig. 5). Less intense immunolabeling was also observed in the RPE and in retinal ganglion cells. At higher magnification, the distribution of PEDF within the IPM can be seen in greater detail (Fig. 6(A) and (B)). PEDF is most abundant surrounding rod and cone outer segments and in the supra-cone space (arrow). Very low levels of PEDF immunolabeling were observed in the extracellular 
A

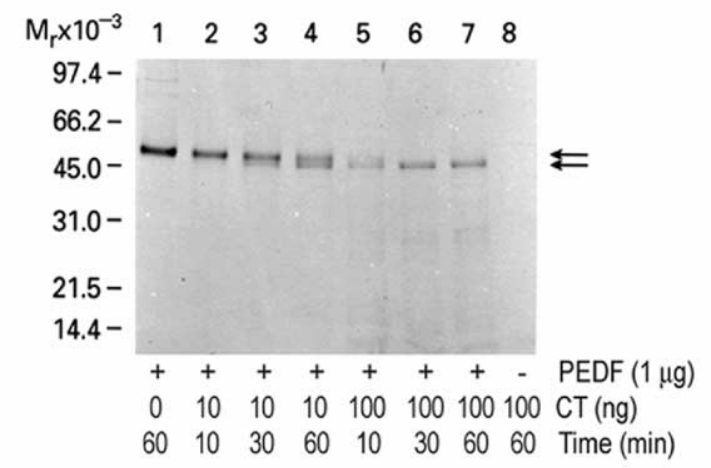

C

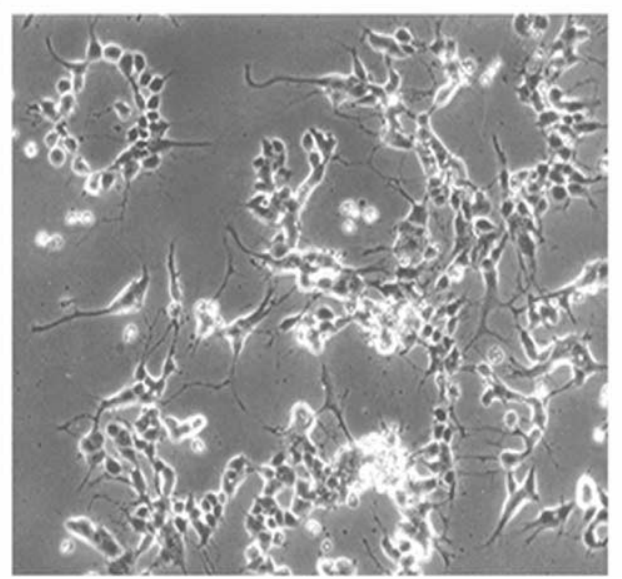

B

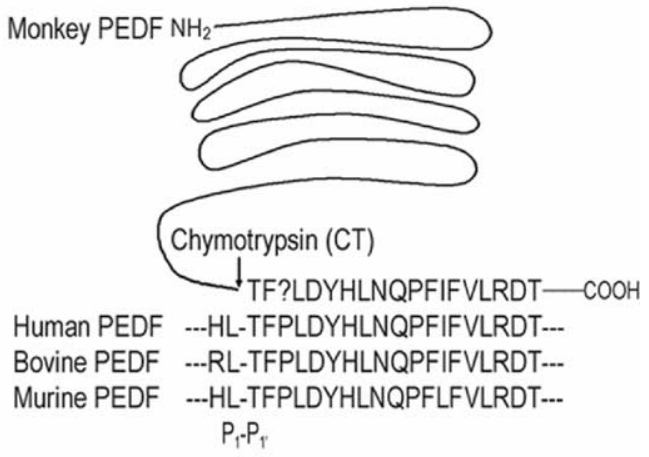

D

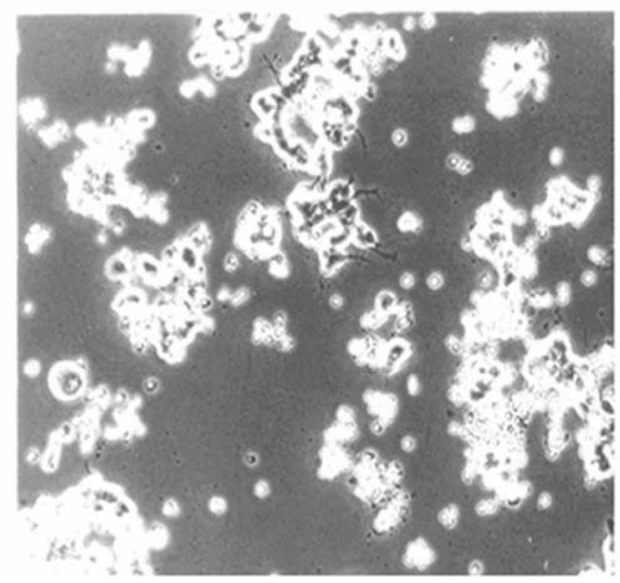

Fig. 3. Characterization of monkey PEDF. (A) Controlled proteolysis of monkey PEDF. Purified protein was incubated with chymotrypsin at $25^{\circ} \mathrm{C}$ in a $15 \mu 1$ reaction containing $20 \mathrm{~mm}$ Tris $\mathrm{HCl}, \mathrm{pH} 7 \cdot 5,150 \mathrm{~mm} \mathrm{NaCl}$, and $1 \mathrm{~mm}$ EDTA, as indicated. The reactions were stopped by addition of SDS-PAGE sample buffer and the products analyzed by SDS/10-20\% PAGE and Coomassie Blue staining. Arrows correspond to migration positions of PEDF substrate and product, and numbers to the left to migration positions of SDS-PAGE molecular weight standards. (B) Scheme of partial amino acid sequence of monkey PEDF and alignment with human, bovine and mouse PEDF sequences. Chymotryptic cleavage between P1 and P1' located towards the carboxy-end of the PEDF molecule, illustrated by an arrow, yields limited products of 46-kDa and 4-kDa. Sequence comparison and alignment using a BLAST program (Altschul et al., 1997) of the 4-kDa product downstream from position P1 of monkey PEDF and GenBank ${ }^{\mathrm{TM}}$ data bases revealed that the highest identity was with human, bovine and mouse PEDF sequences. This region constitutes the signature sequence of serpins, further confirming that the monkey protein is the serpin PEDF. (C) and (D) Biological activity of monkey PEDF. Representative fields of human retinoblastoma Y-79 cells treated with monkey PEDF at 9-day post attachment showing typical morphological differentiation induced by PEDF. (C) $100 \mathrm{ng} \mathrm{ml}^{-1}$ monkey PEDF purified from vitreous plus $125 \mu \mathrm{g}$ ml ${ }^{-1}$ BSA; (D) $125 \mu \mathrm{g} \mathrm{ml}^{-1} \mathrm{BSA}$.

space surrounding the rod and cone inner segments, an area that has been shown previously to contain PEDF receptors (Aymerich et al., 2001). Low to moderate levels of PEDF were also observed in the basal region of the RPE. The lectin PNA, which specifically labels the cone matrix sheath (Fariss et al., 1990), was used to delineate this extracellular domain surrounding cone photoreceptors. PEDF immunolabeling was not detected in the cone matrix sheath but was abundant in the narrow region between the cone outer segment and the cone matrix sheath, as well as the supracone space. Alexa 568 phalloidin was used in doublelabeling studies with antibodies to PEDF to evaluate the intracellular and extracellular distribution of PEDF (Fig. 7(A) and (B)). Given that Phalloidin has binding affinity for filamentous actin, it can be used to demonstrate the dimensions of the RPE actin cytoskeleton (Vaughan and
Fisher, 1987). At high magnification the apical and basal surfaces of the RPE were visible (Fig. 7(B)). The thin line of phalloidin labeling along the basal surface of the RPE showed the basal boundary of these cells. PEDF immunolabeling in this area was clearly intracellular. Very low but detectable PEDF immunolabeling was also observed in Bruch's membrane. The intensity of the fluorescence for PEDF in the RPE decreased sharply at the interface with the apical surface of the RPE. This might be due to melanin present in that area of RPE cells, which can quench fluorescence (personal observations, R. Fariss). Immunohistochemistry of the monkey RPE confirmed specific labeling of PEDF distributed through the RPE cells, although still somehow difficult to distinguish under the area of melanin accumulation (compare Fig. 7(C) and (D)). 
A

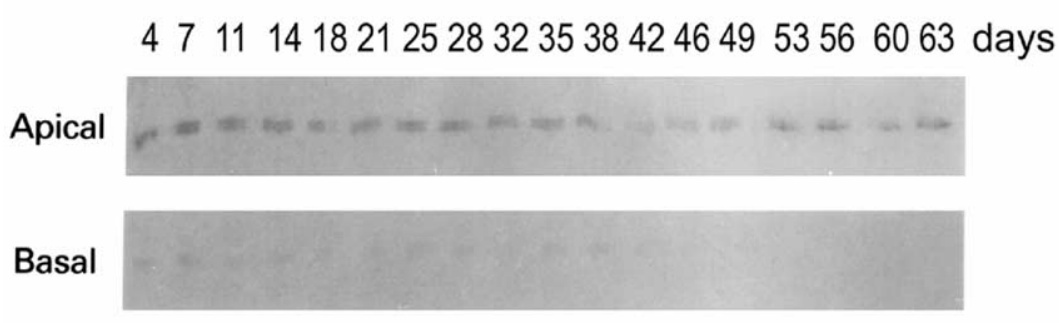

B

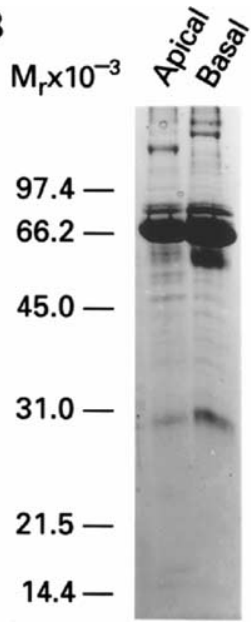

Fig. 4. Directional release of PEDF by polarized RPE cells. Polarized monkey RPE cell cultures were cultivated using permeable supports in serum-containing media. Media were collected from the apical and basal compartments and replaced with fresh media in cycles of alternating 4 and 3 days after a stable, confluent monolayer was attained. Collected apical and basal media from each cycle were analyzed for PEDF content. (A) Immunoblot analysis with antiserum Ab-rPEDF. The numbers at the top of each lane correspond to days post-attaining confluence. PEDF in each medium was quantified by densitometric analysis of the immunoreactivity and the apical:basal ratio of PEDF secretion was determined from these values for each time point. The apical:basal ratio started with 3.8:1 and increased with time to $>28: 1$. (B) Total protein analysis of the media by SDS-PAGE and Coomassie Blue staining. Replicate apical and basal samples in panel A at 42 days post-attaining confluence are shown (as indicated). Migration positions of SDS-PAGE molecular weight standards are to the left.

\section{Discussion}

The present study indicates that in the monkey eye the RPE preferentially releases the mature PEDF protein from its apical side, and suggests that the RPE targets PEDF towards the photoreceptors of the neural retina. This conclusion is supported by several facts: (1) PEDF accumulates preferentially in the apical media of polarized RPE cell cultures; (2) IPM lavages have high concentrations of soluble PEDF; and (3) strong and specific immunoreactivity for PEDF is detected to the apical side of the RPE in the IPM area surrounding the photoreceptor rods and cones in the retina. Although the steady-state polarity of PEDF from the cultured RPE cells indicates that secretion of PEDF from the apical side of RPE cells is preferred over the basal side, we cannot discard the possibilities that (1) the basal side could have proteases resulting in degradation of the PEDF released into the basal compartment; and (2) upon basally-directed secretion, PEDF could bind to components of extracellular matrix normally found in basement membranes (such as collagens, glycosaminoglycans) preventing diffusion of the secreted PEDF into the medium. Nonetheless, the distribution of PEDF in the RPE and IPM in vivo is in agreement with a preferred apicolateral secretion by the RPE. It is clear that further information is required to confirm intracellular targeting of the precursor PEDF to the apical membrane of polarized RPE cells. However, the present results demonstrate that the polarized RPE releases mature PEDF protein toward the adjacent high affinity binding sites in photoreceptors that are available to interact with the secreted PEDF ligand (Aymerich et al., 2001), and suggest a role of PEDF in a paracrine relation between the RPE and the photoreceptors. These observations are consistent with the known trophic function of
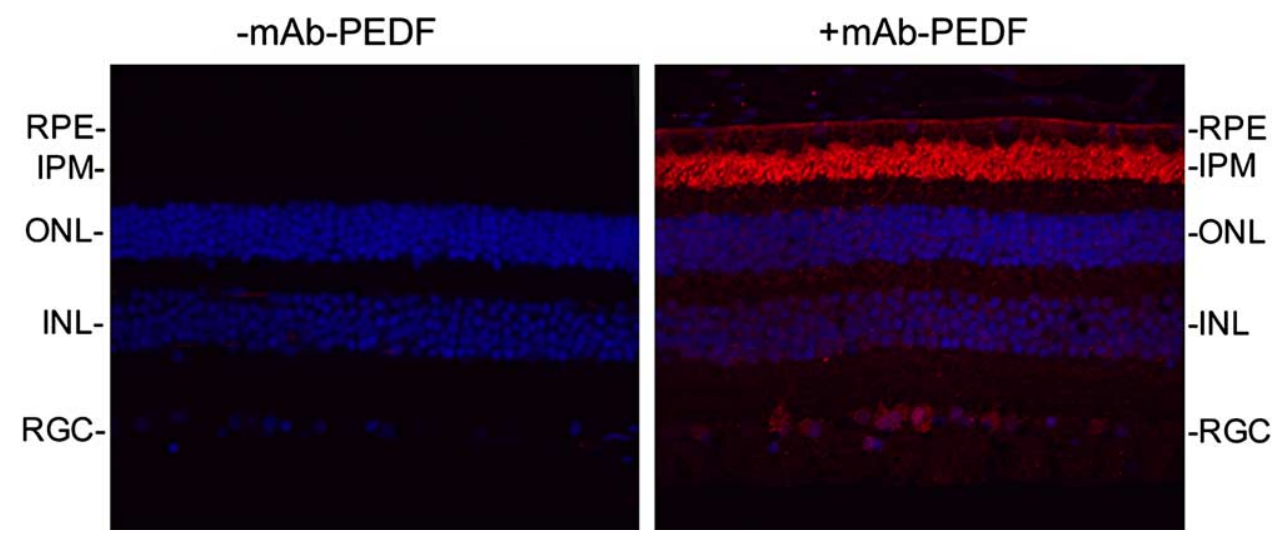

Fig. 5. Confocal immunofluorescence localization of PEDF in monkey retina. PEDF immunolabeling, located in the layers containing photoreceptors outer segments (OS) and inner segments (IS), is red. Nuclei in the neural retina and RPE, labeled with the DNA-binding fluorescent dye DAPI, are in blue. PEDF protein is abundant in the IPM. Left panel, minus monoclonal antibody to PEDF; right panel, plus monoclonal antibody to PEDF. 
A

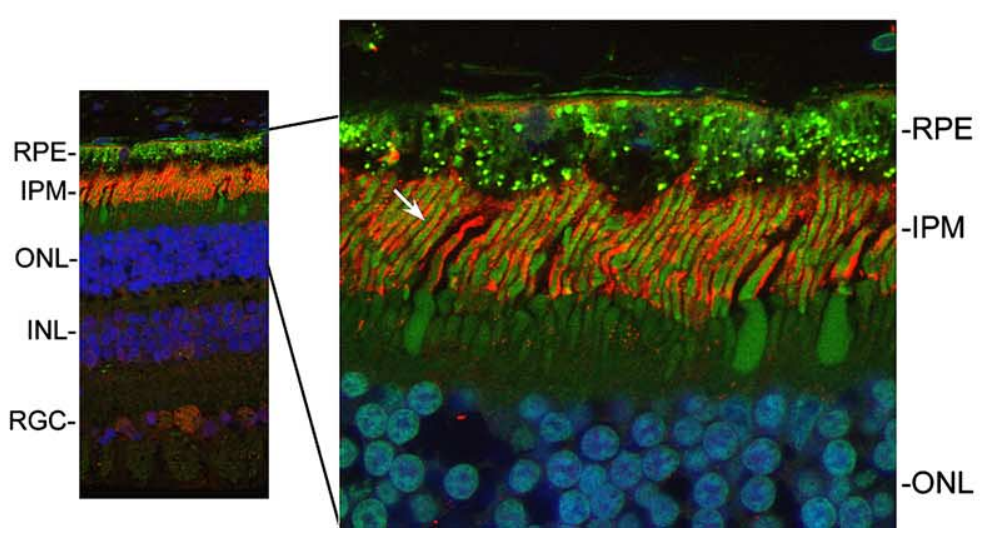

B

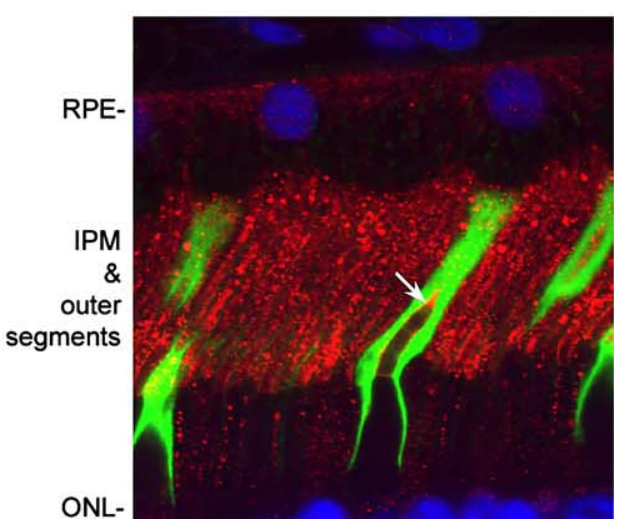

Fig. 6. PEDF surrounds the outer segments of the photoreceptors. (A) Immunofluorescence of the monkey retina visualized by confocal microcopy. Autofluorescence, typical of rod outer segments of photoreceptors, is visualized in green, PEDF immunoreactivity (PEDF Cy3), and DAPI in blue. At a higher magnification, PEDF immunolabeling was visible in the IPM surrounding rod and cone OS (arrow). (B) Immunofluorescence of the monkey retina to visualize cone matrix sheath with PNA and PEDF with monoclonal antibody to PEDF. PNA biotin/Strept Cy5 is in green, PEDF/GAM 488 in red, and DAPI in blue. PEDF immunolabeling was detected surrounding the cone outer sheaths (arrow). Moderately high levels of autofluorescence were visible in Bruch's membrane and the RPE in negative controls. Retinal vessels in the inner retina were also fluorescently labeled in the negative control (no primary).

the RPE in the maintenance of the photoreceptors and point to a role for PEDF in normal eye functioning. In addition, PEDF may be employed as a marker for RPE polarization in culture.

Several lines of evidence point to the importance of PEDF for the retina and its exploitation as a therapeutic agent for retinal diseases triggered by photoreceptor degenerations and abnormal neovascularization. To our knowledge this is the first presentation of data providing comprehensive information on levels and concentrations of
PEDF in ocular compartments of the primate eye. The levels of PEDF in monkey vitreous are within the range of those reported previously in humans, e.g. $23.7 \pm 0.7 \mathrm{nM}$ (Spranger et al., 2001), $1.7 \pm 0.22 \mu \mathrm{g} \mathrm{ml}^{-1}$ (Ogata et al., 2002), with the exception of one, about $16 \mu \mathrm{g} \mathrm{ml}^{-1}$ (Holekamp et al., 2002). It is expected that the determinations of PEDF concentrations in the monkey IPM and aqueous are also within the range of those in their human counterparts. In the monkey eye, the concentrations of soluble PEDF in IPM are 7- and 25-fold higher than in vitreous and aqueous,
A

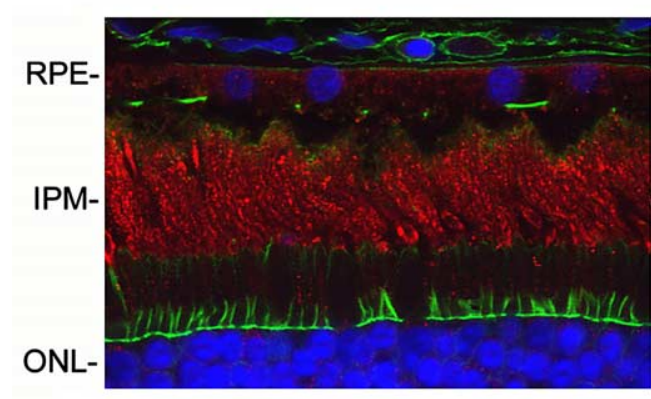

C

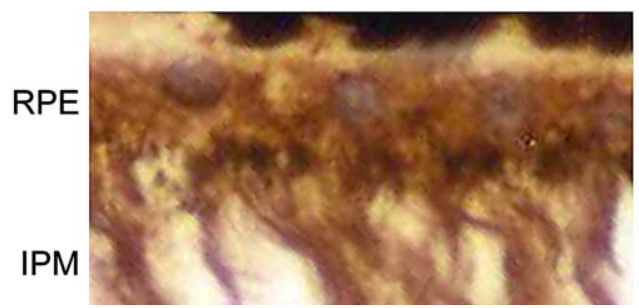

B

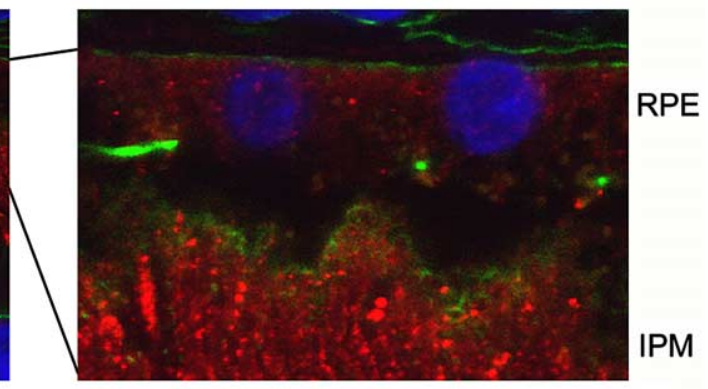

D

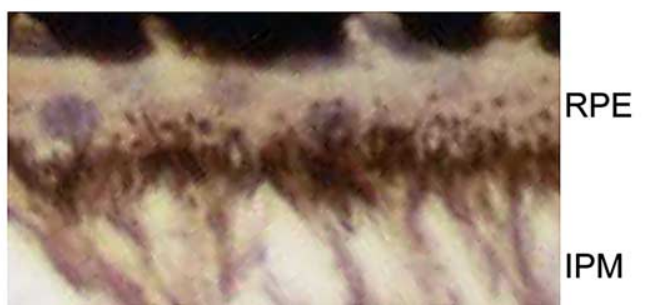

Fig. 7. Intracellular distribution of PEDF in RPE cells in situ. Panels A and B show immunofluorescence of monkey retina to visualize the cell boundary with phalloidin, an actin marker. Monoclonal antibody to PEDF was used. PEDF/GAM 488 (red), Phalloidin $568 \mathrm{~nm}$ (green), DAPI (blue). Panel B is at higher magnification than the panel A. Panels $\mathrm{C}$ and D show immunohistochemistry of monkey retina to visualize PEDF in the RPE cells. Panel C shows crosssections of the RPE-IPM area showing specific immunoreactivity to the polyclonal antiserum to rhuPEDF, Ab-rPEDF, in the RPE region, and panel D shows negative immunoreactivity controls with preabsorbed Ab-rPEDF with PEDF antigen. 
respectively, similar to the concentrations previously reported in the bovine eye ( $\mathrm{Wu}$ et al., 1995; $\mathrm{Wu}$ and Becerra, 1996). These values represent the basal levels of this interesting neurotrophic and antiangiogenic factor in the primate eye. Changes in PEDF levels in the eye with angiogenic retina or retinal degenerations may contribute to the development or progression of these disorders. Evidence for an association between decreased PEDF and induced or diseased-related angiogenesis (Spranger et al., 2001; Gao et al., 2002; Ogata et al., 2002; Holekamp et al., 2002) makes the present information on physiological PEDF levels more valuable for diagnostic and therapeutic studies.

Our immunolabeling studies of monkey retina demonstrate the localization of PEDF in the IPM, and at low to moderate levels in the RPE cytoplasm and retinal ganglion cells. These results were obtained with a monoclonal antiPEDF antibody that recognized a single band on westerns of monkey retinal and RPE extracts. Using the same monoclonal anti-PEDF antibody, Karakousis et al. (2001) reported PEDF-immunolabeling in the IPM of adult human retina. However, they were unable to detect PEDFimmunolabeling in the RPE due to high levels of lipofuscin autofluorescence in their samples. Using a polyclonal antibody to PEDF, they reported widespread distribution of this protein in photoreceptor inner and outer segments, rod nuclei, rod and cone synapse, a subset of cells in the inner nuclear layer and ganglion cell layer. However, this polyclonal antibody was shown by these authors to label multiple bands migrating slower and faster than PEDF on westerns of human retinal extracts. PEDF localization in the IPM has been reported previously in weanling rats (Becerra et al., 1999). Thus, the distribution of PEDF in the monkey IPM is in agreement with those previously reported for other species, and the present study provides new information on the distribution of PEDF in the RPE.

We have established a polarized RPE cell culture that expresses, synthesizes and produces high levels of PEDF. Expressed in comparative quantitative terms, $2.6 \mathrm{mg}$ of purified monkey PEDF protein can be obtained from one liter of RPE conditioned media, vitreous from 2167 eyes or IPM lavage from 15600 eyes. The average concentration of PEDF protein in the culture medium of RPE cells attached to plastic after 8 days in culture reached $6.5 \mu \mathrm{g} \mathrm{ml}^{-1}$, a high value obtained from primary cultures. Considering the number of cells cultured we estimate that the media has $1 \cdot 1 \mathrm{pg}$ of PEDF per RPE cell. Note that in polarized cells, the steady-state levels at the apical side were maintained during the course of the study of 63 days. Thus, very stringent conditions for culturing these cells are optimal for high expression of the $P E D F$ gene and secretion of the protein.

The data reported here also provide biochemical, structural, immunological and biological evidence that the protein purified from monkey sources is PEDF. These observations suggest that this protein is the monkey homologue of the previously described human, bovine and mouse PEDF that contains the structural determinants for biological function. Because both humans and monkeys are within the same taxonomic order, it is expected that monkey and human PEDF share a high degree of sequence similarity. In this regard, the monkey PEDF shares with the human the lack of a tryptic site in the serpin-exposed loop, which is present in the bovine PEDF. The tryptic recognition site in the bovine PEDF is an arginine in position $\mathrm{P} 2$, which in the human PEDF is occupied by a histidine and is likely to be conserved in the monkey, thus losing the tryptic site. However, it will be necessary to isolate and sequence cDNA clones for monkey PEDF to provide evidence on the degree of identity between the fulllength monkey and human PEDF, as well as among other species.

Our data offer interesting possibilities on regulation of PEDF. The polarization of RPE may be an important mechanism to control PEDF secretion, as is the rate of $P E D F$ gene expression and PEDF protein synthesis. The data obtained so far on the action of PEDF on the retina may have not considered the polar secretion of PEDF by RPE. Regulation of RPE polarization by extracellular stimulus or signaling molecules from the choroid or neural retina, may affect the levels of PEDF secreted by the RPE. The fact that extracellular molecules or oxygen can switch the polarization of RPE from apical to basal and vice versa (Mousa et al., 1999; Blaauwgeers et al., 1999; Koh, 2000) suggests the possibility of the existence of a stimulus that could specifically target the change of polarity for PEDF secretion to the choroid (basal side) or increase the secretion to the IPM (apical side), thus increasing PEDF intrinsic activities for the choroid or neural retina. Another possibility for regulation might be at the secretion level. In this regard the apicolateral secretion of PEDF and specific distribution within the IPM entail the possibility of corelease and targeting in association with the glycosaminoglycans also synthesized by the RPE. For example, the non-sulfated glycosaminoglycan hyaluronan, with binding affinity for PEDF (Becerra et al., 1998), is also secreted in a polar fashion to the apical side of human fetal RPE cells in culture and is distributed to the IPM (deS Senanayake, 2001), suggesting an association of these two molecules intracellularly and secretion in concert. Thus, the loss or increase of glycosaminoglycan expression could affect the amount of PEDF secretion. In the IPM, the glycosaminoglycans may serve as a depository matrix for PEDF located in proximity to the PEDF-receptor in photoreceptor cells, and selective glycosaminoglycans can be positive modulators of the interactions between PEDF and its receptors (Alberdi et al., 2003) by increasing its activity. Finally, the structural determinants for secretion, identified in a recent mutagenic study at a region of the serpin exposed loop of human PEDF (Shao et al., 2003), would recognize the secretory components polarized to the apical membrane of the RPE cells. The existence of natural PEDF variants with mutations or deletions of this region would 
have a decrease in secretion rate, resulting in a decrease in antiangiogenic and neurotrophic activity in the IPM. These possibilities are discussed under the consideration that some of them may have important implications if PEDF, or molecules derived from it, are to be used in the future as therapeutic agents.

In conclusion, the observation that RPE cells secrete PEDF in a polarized fashion towards the neural retina may represent a mechanism to prevent damage to the adjacent fragile retinal tissue. This is consistent with the known trophic function of the RPE in the maintenance of the photoreceptors and points to a role for PEDF in normal eye functioning. However, loss of polarity of PEDF production may play a role in the pathogenesis of retinal degenerations or/and choroidal or retinal neovascularization.

\section{Acknowledgements}

We thank Dr Steve Bernstein for help in the dissection of the ocular tissues from monkey eyes, Dr Vicente Notario for help in the biological assay, Ms Patricia Spinella for performing amino acid sequencing, and Drs Barbara Wiggert and Juan Amaral for interesting discussions and proof reading the manuscript.

\section{References}

Adler, A., 1989. Selective presence of acid hydrolases in the interphotoreceptor matrix. Exp. Eye Res. 49, 1067-1077.

Alberdi, E., Hyde, C.C., Becerra, S.P., 1998. Pigment epithelium-derived factor (PEDF) binds to glycosaminoglycans: analysis of the binding site. Biochemistry 37, 10643-10652.

Alberdi, E., Aymerich, M.S., Becerra, S.P., 1999. Binding of pigment epithelium-derived factor (PEDF) to retinoblastoma cells and cerebellar granule neurons. J. Biol. Chem. 274, 31605-31612.

Alberdi, E.M., Weldon, J.E., Becerra, S.P., 2003. Glycosaminoglycans in human retinoblastoma cells: heparan sulfate, a modulator of the pigment epithelium-derived factor-receptor interactions. Biochemistry $4,1$.

Altschul, S.F., Madden, T.L., Schäffer, A.A., Zhang, J., Zhang, Z., Miller, W., Lipman, D.J., 1997. Gapped BLAST and PSI-BLAST: a new generation of protein database search programs. Nucleic Acids Res. 25, 3389-3402.Review.

Aymerich, M.S., Alberdi, E.M., Martinez, A., Becerra, S.P., 2001. Evidence for pigment epithelium-derived factor receptors in the neural retina. Invest. Ophthalmol. Vis. Sci. 42, 3287-3293.

Becerra, S.P., Alberdi, E., Martinez, A., Montuenga, L.M., Cayouette, M., Gravel, C., 1999. Pigment epithelium-derived factor (PEDF) in the retina: Protective effect against photoreceptor cell degeneration. In: Hollyfield, J.G., et al. (Eds.), In Retinal Degenerative Diseases and Experimental Therapy, Kluwer Academic/Plenum, New York, pp. 519-526.

Becerra, S.P., Hollyfield, J.G., Iza-Azcarate, I., Perez-Mediavilla, L.A., 1998. Pigment epithelium-derived factor (PEDF) has binding affinity for hyaluronan. Invest. Ophthalmol. Vis. Sci. 40, 42.

Becerra, S.P., Palmer, I., Kumar, A., Steele, F., Shiloach, J., Notario, V., Chader, G.J., 1993. Overexpression of pigment epithelium-derived factor in Escherichia coli. A functionally active neurotrophic factor. J. Biol. Chem. 268, 23148-23156.

Becerra, S.P., Sagasti, A., Spinella, P., Notario, V., 1995. Pigment epithelium-derived factor behaves like a noninhibitory serpin. Neurotrophic activity does not require the serpin reactive loop. J. Biol. Chem. 270, 25992-25999.

Becerra, S.P., 1997. Structure-function studies on PEDF. A noninhibitory serpin with neurotrophic activity. Adv. Exp. Med. Biol. 425, 223-237.

Bilak, M.M., Becerra, S.P., Vincent, A.M., Moss, B.H., Aymerich, M.S., Kuncl, R.W., 2002. Identification of the neuroprotective molecular region of pigment epithelium-derived factor and its binding sites on motor neurons. J. Neurosci. 22, 9378-9386.

Blaauwgeers, H.G., Holtkamp, G.M., Rutten, H., Witmer, A.N., Koolwijk, P., Partanen, T.A., Alitalo, K., Kroon, M.E., Kijlstra, A., van Hinsbergh, V.W., Schlingemann, R.O., 1999. Polarized vascular endothelial growth factor secretion by human retinal pigment epithelium and localization of vascular endothelial growth factor receptors on the inner choriocapillaris. Evidence for a trophic paracrine relation. Am. J. Pathol. $155,421-428$.

Bouck, N., 2002. PEDF: anti-angiogenic guardian of ocular function. Trends Mol. Med., 330-334. Review.

Cao, W., Tombran-Tink, J., Elias, R., Sezate, S., Mrazek, D., McGinnis, J.F., 2001. In vivo protection of photoreceptors from light damage by pigment epithelium-derived factor. Invest. Ophthalmol. Vis. Sci. 42, $1646-1652$.

Cayouette, M., Smith, S.B., Becerra, S.P., Gravel, C., 1999. Pigment epithelium-derived factor delays the death of photoreceptors in mouse models of inherited retinal degenerations. Neurobiol. Dis. 6, 523-532.

Crawford, S.E., Stellmach, V., Ranalli, M., Huang, X., Huang, L., Volpert, O., De Vries, G.H., Abramson, L.P., Bouck, N., 2001. Pigment epithelium-derived factor (PEDF) in neuroblastoma: a multifunctional mediator of Schwann cell antitumor activity. J. Cell. Sci. 114, 4421-4428.

Dawson, D.W., Volpert, O.V., Gillis, P., Crawford, S.E., Xu, H., Benedict, W., Bouck, N.P., 1999. Pigment epithelium-derived factor: a potent inhibitor of angiogenesis. Science 285, 245-248.

deS Senanayake, P., Calabro, A., Nishiyama, K., Hu, J.G., Bok, D., Hollyfield, J.G., 2001. Glycosaminoglycan synthesis and secretion by the retinal pigment epithelium: polarized delivery of hyaluronan from the apical surface. J. Cell. Sci. 114, 199-205.

Duh, E.J., Yang, H.S., Suzuma, I., Miyagi, M., Youngman, E., Mori, K., Katai, M., Yan, L., Suzuma, K., West, K., Davarya, S., Tong, P., Gehlbach, P., Pearlman, J., Crabb, J.W., Aiello, L.P., Campochiaro, P.A., Zack, D.J., 2002. Pigment epithelium-derived factor suppresses ischemia-induced retinal neovascularization and VEGF-induced migration and growth. Invest. Ophthalmol. Vis. Sci. 43, 821-829.

Dunn, K.C., Marmorstein, A.D., Bonilha, V.L., Rodriguez-Boulan, E., Giordano, F., Hjelmeland, L.M., 1998. Use of the ARPE-19 cell line as a model of RPE polarity: basolateral secretion of FGF5. Invest. Ophthalmol. Vis. Sci. 39, 2744-2749.

Fariss, R.N., Anderson, D.H., Fisher, S.K., 1990. Comparison of photoreceptor-specific matrix domains in the cat and monkey retinas. Exp. Eye Res. 51, 473-485.

Fariss, R.N., Apte, S.S., Olsen, B.R., Iwata, K., Milam, A.H., 1997. Tissue inhibitor of metalloproteinases- 3 is a component of Bruch's membrane of the eye. Am. J. Pathol. 150, 323-328.

Gao, G., Li, Y., Fant, J., Crosson, C.E., Becerra, S.P., Ma, J.X., 2002. Difference in ischemic regulation of vascular endothelial growth factor and pigment epithelium-derived factor in brown norway and sprague dawley rats contributing to different susceptibilities to retinal neovascularization. Diabetes 51, 1218-1225.

Holekamp, N.M., Bouck, N., Volpert, O., 2002. Pigment epitheliumderived factor is deficient in the vitreous of patients with choroidal neovascularization due to age-related macular degeneration. Am. J. Ophthalmol. 134, 220-227. 
Holtkamp, G.M., Van Rossem, M., de Vos, A.F., Willekens, B., Peek, R., Kijlstra, A., 1998. Polarized secretion of IL- 6 and IL- 8 by human retinal pigment epithelial cells. Clin. Exp. Immunol. 112, 34-43.

Houenou, L.J., D’Costa, A.P., Li, L., Turgeon, V.L., Enyadike, C., Alberdi, E., Becerra, S.P., 1999. Pigment epithelium-derived factor promotes the survival and differentiation of developing spinal motor neurons. J. Comp. Neurol. 412, 506-514.

Karakousis, P.C., John, S.K., Behling, K.C., Surace, E.M., Smith, J.E., Hendrickson, A., Tang, W.X., Bennett, J., Milam, A.H., 2001. Localization of pigment epithelium derived factor (PEDF) in developing and adult human ocular tissues. Mol. Vis. 7, 154-163.

Koh, S.M., 2000. VIP enhances the differentiation of retinal pigment epithelium in culture: from cAMP and pp60(c-src) to melanogenesis and development of fluid transport capacity. Prog. Retin. Eye Res. 19, $669-688$.

Hale, I.L., Matsumoto, B., 1993. Resolution of subcellular detail in thick tissue sections: immunohistochemical preparation and fluorescence confocal microscopy. Meth. Cell. Biol., 289-324. Review.

Montuenga, L.M., Martinez, A., Miller, M.J., Unsworth, E.J., Cuttitta, F., 1997. Expression of adrenomedullin and its receptor during embryogenesis suggests autocrine or paracrine modes of action. Endocrinology 138, 440-451.

Meyer, C., Notari, L., Becerra, S.P., 2002. Mapping the type I collagenbinding site on pigment epithelium-derived factor. Implications for its antiangiogenic activity. J. Biol. Chem. 277, 45400-454007.

Mousa, S.A., Lorelli, W., Campochiaro, P.A., 1999. Role of hypoxia and extracellular matrix-integrin binding in the modulation of angiogenic growth factors secretion by retinal pigmented epithelial cells. J. Cell. Biochem. 74, 135-143.

Ogata, N., Nishikawa, M., Nishimura, T., Mitsuma, Y., Matsumura, M., 2002. Unbalanced vitreous levels of pigment epithelium-derived factor and vascular endothelial growth factor in diabetic retinopathy. Am. J. Ophthalmol. 134, 348-353.

Ortego, J., Escribano, J., Becerra, S.P., Coca-Prados, M., 1996. Gene expression of the neurotrophic pigment epithelium-derived factor in the human ciliary epithelium. Synthesis and secretion into the aqueous humor. Invest. Ophthalmol. Vis. Sci. 37, 2759-2767.

Padgett, L.C., Lui, G.M., Werb, Z., LaVail, M.M., 1997. Matrix metalloproteinase-2 and tissue inhibitor of metalloproteinase-1 in the retinal pigment epithelium and interphotoreceptor matrix: vectorial secretion and regulation. Exp. Eye Res. 64, 927-938.

Perez-Mediavilla, L.A., Chew, C., Campochiaro, P.A., Nickells, R.W., Notario, V., Zack, D.J., Becerra, S.P., 1998. Sequence and expression analysis of bovine pigment epithelium-derived factor. Biochim. Biophys. Acta 1398, 203-214.

Pfeffer, B.A., 1990. Improved methodology for cell culture of human and monkey retinal pigment epithelium. In: Osborne, N.N., Chader, G.J.
(Eds.), Progress in Retinal Research, vol. 10. Pergamon Press, Oxford, UK, pp. 251-291.

Pfeffer, B., Wiggert, B., Lee, L., Zonnenberg, B., Newsome, D., Chader, G. 1983. The presence of a soluble interphotoreceptor matrix retinolbinding protein (IRBP) in the retinal interphotoreceptor space. J. Cell. Physiol. 117, 333-341.

Pignolo, R.J., Cristofalo, V.J., Rotenberg, M.O., 1993. Senescent WI-38 cells fail to express EPC-1, a gene induced in young cells upon entry into the G0 state. J. Biol. Chem. 268, 8949-8957.

Shao, H., Schvartz, I., Shatiel, S., 2003. Secretion of pigment epitheliumderived factor: mutagenic study. Eur. J. Biochem. 270, 822-831.

Spranger, J., Osterhoff, M., Reimann, M., Mohlig, M., Ristow, M., Francis, M.K., Cristofalo, V., Hammes, H.P., Smith, G., Boulton, M., Pfeiffer, A.F., 2001. Loss of the antiangiogenic pigment epithelium-derived factor in patients with angiogenic eye disease. Diabetes 50, 2641-2645.

Steele, F.R., Chader, G.J., Johnson, L.V., Tombran-Tink, J., 1993. Pigment epithelium-derived factor: neurotrophic activity and identification as a member of the serine protease inhibitor gene family. Proc. Nat. Acad. Sci. USA 90, 1526-1530.

Stellmach, V.V., Crawfordm, S.E., Shou, W., Bouck, N., 2001. Prevention of ischemia-induced retinopathy by the natural ocular antiangiogenic agent pigment epithelium-derieved factor. Proc. Nat. Acad. Sci. USA 98, 2593-2597.

Stratikos, E., Alberdi, E., Gettins, P.G., Becerra, S.P., 1996. Recombinant human pigment epithelium-derived factor (PEDF): characterization of PEDF overexpressed and secreted by eukaryotic cells. Protein Sci. 5, $2575-2582$.

Tombran Tink, J., Chader, G.G., Johnson, L.V., 1991. PEDF: a pigment epithelium-derived factor with potent neuronal differentiative activity. Exp. Eye Res. 53, 411-414.

Tombran-Tink, J., Shivaram, S.M., Chader, G.J., Johnson, L.V., Bok, D., 1995. Expression, secretion, and age-related downregulation of pigment epithelium-derived factor, a serpin with neurotrophic activity. J. Neurosci. 15, 4992-5003.

Vaughan, D.K., Fisher, S.K., 1987. The distribution of F-actin in cells isolated from vertebrate retinas. Exp. Eye Res. 44, 393-406.

Wu, Y.Q., Notario, V., Chader, G.J., Becerra, S.P., 1995. Identification of pigment epithelium-derived factor in the interphotoreceptor matrix of bovine eyes. Protein Exp. Purif. 6, 447-456.

Wu, Y.Q., Becerra, S.P., 1996. Proteolytic activity directed toward pigment epithelium-derived factor in vitreous of bovine eyes. Implications of proteolytic processing. Invest. Ophthalmol. Vis. Sci. 37, 1984-1993.

Wong, P., Pfeffer, B.A., Bernstein, S.L., Chambers, M.L., Chader, G.J., Zakeri, Z.F., Wu, Y.Q., Wilson, M.R., Becerra, S.P., 2000. Clusterin protein diversity in the primate eye. Mol. Vis. 6, 184-191. 